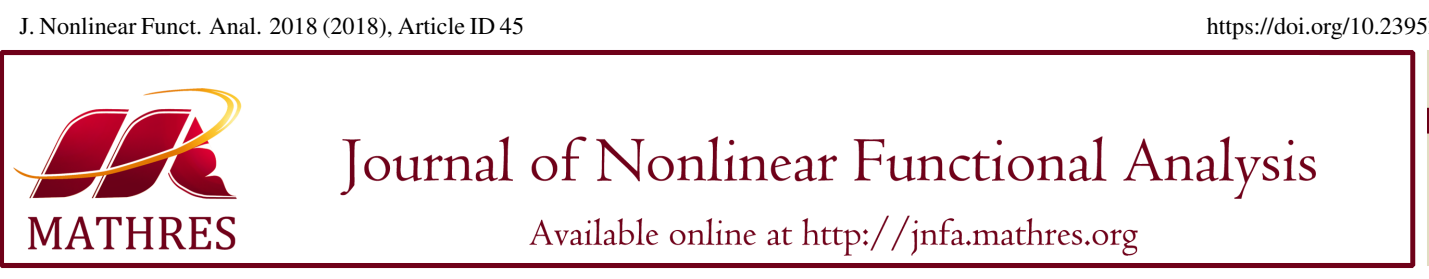

https://doi.org/10.23952/jnfa.2018.45

\title{
A UNIFIED APPROACH TO SOME INEQUALITIES FOR GENERALIZED CONVEX FUNCTIONS ON FRACTAL SETS AND THEIR APPLICATIONS
}

\author{
WENBING SUN \\ School of Science, Shaoyang University, Shaoyang 422000, Hunan, China
}

\begin{abstract}
In this paper, a general local fractional integral identity on fractal set $\mathbb{R}^{\alpha}(0<\alpha \leq 1)$ is established. We obtain some general local fractional integral inequalities for twice differentiable generalized convex functions. Based on these results, we derive some special inequalities. Some applications to error estimations for numerical integration and to special means are also given.
\end{abstract}

Keywords. Fractal space; Local fractional integral; Hadamard's inequality; Generalized convex function, Integral inequality.

2010 Mathematics Subject Classification. 26D15, 26A51, 26D10.

\section{INTRODUCTION}

Let $f: I \subseteq \mathbb{R} \rightarrow \mathbb{R}$ be a convex function and $a, b \in I$ with $a<b$. Then, the following inequality holds

$$
f\left(\frac{a+b}{2}\right) \leq \frac{1}{b-a} \int_{a}^{b} f(x) d x \leq \frac{f(a)+f(b)}{2},
$$

which is well known as Hermite-Hadamard's inequality.

Let $f:[a, b] \rightarrow \mathbb{R}$ be a four times continuously differentiable mapping on $(a, b)$,

$$
\left\|f^{(4)}\right\|_{\infty}=\sup _{x \in(a, b)}\left|f^{(4)}\right|<\infty .
$$

Then

$$
\left|\frac{1}{3}\left[\frac{f(a)+f(b)}{2}+2 f\left(\frac{a+b}{2}\right)\right]-\frac{1}{b-a} \int_{a}^{b} f(x) d x\right| \leq \frac{1}{2880}\left\|f^{(4)}\right\|_{\infty}(b-a)^{4} .
$$

Inequality (1.2) is named the Simpson's inequality, which is one of the best known inequalities.

In recent years, Hadamard's type inequalities and Simpson's type inequality were generalized, improved and refined by many researchers for different convexity. For more recent results, one can see [1]-[10] and the references cited therein.

In [11], Sarikaya and Aktan established midpoint inequalities, trapezoid inequalities and Simpson's inequalities for functions whose second derivatives absolute values are convex, and established the following inequalities.

E-mail address: swb0520@163.com.

Received October 29, 2018; Accepted December 10, 2018.

(c)2018 Journal of Nonlinear Functional Analysis 
Theorem 1.1. Let $I \subset \mathbb{R}$ be an open interval, and $a, b \in I$ with $a<b$. Let $f: I \rightarrow \mathbb{R}$ be a twice differentiable mapping such that $f^{\prime \prime}$ is integrable and $0 \leq \lambda \leq 1$. If $\left|f^{\prime \prime}\right|$ is a convex on $[a, b]$, then the following inequalities hold:

$$
\begin{aligned}
& \left|(\lambda-1) f\left(\frac{a+b}{2}\right)-\lambda \frac{f(a)+f(b)}{2}+\frac{1}{(b-a)} \int_{a}^{b} f(x) d x\right| \\
& \leq \begin{cases}\frac{(b-a)^{2}}{12}\left[\left(\lambda^{4}+(1+\lambda)(1-\lambda)^{3}+\frac{5 \lambda-3}{4}\right)\left|f^{\prime \prime}(a)\right|\right. & \\
\left.+\left(\lambda^{4}+(2-\lambda) \lambda^{3}+\frac{1-3 \lambda}{4}\right)\left|f^{\prime \prime}(b)\right|\right], & 0 \leq \lambda \leq \frac{1}{2}, \\
\frac{(b-a)^{2}(3 \lambda-1)}{48}\left(\left|f^{\prime \prime}(a)\right|+\left|f^{\prime \prime}(b)\right|\right), & \frac{1}{2} \leq \lambda \leq 1 .\end{cases}
\end{aligned}
$$

Recently, fractional calculus received significantly remarkable attention. Particulary, Yang [12, 13] systematically stated the theory of local fractional calculus on fractal sets. Many researchers extended the research on integral inequalities to fractal space, see [14]-[23] and the references therein. In [23], Mo, Sui and Yu introduced the definition of the generalized convex function on fractal sets, and proved the following generalized Hermite-Hadamard's inequality.

Theorem 1.2. (Generalized Hermite-Hadamard's inequality) Let $f(x) \in I_{x}^{(\alpha)}[a, b]$ be a generalized convex function on $[a, b], a<b$. Then

$$
f\left(\frac{a+b}{2}\right) \leq \frac{\Gamma(1+\alpha)}{(b-a)^{\alpha}} a_{b}^{(\alpha)} f(x) \leq \frac{f(a)+f(b)}{2^{\alpha}} .
$$

In order to give a unified approach to study the trapezoid inequality, the midpoint inequality and the Simpson's inequality for twice differentiable generalized convex functions on fractal sets, we will establish some general local fractional integral inequalities for functions whose absolute values of twice derivatives are generalized convex functions. Based on these inequalities, we will derive some special inequalities, which includes the midpoint inequality, the trapezoid inequality and the Simpson's inequality. We mainly improve the results announced in [11]. Finally, some applications for numerical integrations involving local fractional integral and some $\alpha$-type special means are also provided.

\section{PRELIMINARIES}

Let $\mathbb{R}^{\alpha}$ be the $\alpha$-type set of the real line numbers, where $0<\alpha \leq 1$. This set $\mathbb{R}^{\alpha}$ is called a fractional set. The set $\mathbb{R}$ is called base set of fractional set, see $[12,13]$ and the references therein. A fractal field of real line numbers is any fractional set of objects together with two operations, addition and multiplication, where the operations satisfy

If $a^{\alpha}, b^{\alpha}, c^{\alpha} \in \mathbb{R}^{\alpha}$, then

(1) $a^{\alpha}+b^{\alpha} \in \mathbb{R}^{\alpha}, a^{\alpha} b^{\alpha} \in \mathbb{R}^{\alpha}$,

(2) $a^{\alpha}+b^{\alpha}=b^{\alpha}+a^{\alpha}=(a+b)^{\alpha}=(b+a)^{\alpha}$,

(3) $a^{\alpha}+\left(b^{\alpha}+c^{\alpha}\right)=(a+b)^{\alpha}+c^{\alpha}$,

(4) $a^{\alpha} b^{\alpha}=b^{\alpha} a^{\alpha}=(a b)^{\alpha}=(b a)^{\alpha}$,

(5) $a^{\alpha}\left(b^{\alpha} c^{\alpha}\right)=\left(a^{\alpha} b^{\alpha}\right) c^{\alpha}$,

(6) $a^{\alpha}\left(b^{\alpha}+c^{\alpha}\right)=a^{\alpha} b^{\alpha}+a^{\alpha} c^{\alpha}$, 
(7) $a^{\alpha}+0^{\alpha}=0^{\alpha}+a^{\alpha}=a^{\alpha}, a^{\alpha} 1^{\alpha}=1^{\alpha} a^{\alpha}=a^{\alpha}$.

Now, we state the definitions of the local fractional derivative and local fractional integral on $\mathbb{R}^{\alpha}$ as follows.

Definition 2.1. $[12,13]$ A non-differentiable function $f: \mathbb{R} \rightarrow \mathbb{R}^{\alpha}, x \rightarrow f(x)$ is said to be local fractional continuous at $x_{0}$, if, for any $\varepsilon>0$, there exists $\delta>0$ such that $\left|f(x)-f\left(x_{0}\right)\right|<\varepsilon^{\alpha}$ for $\left|x-x_{0}\right|<\delta$, where $\varepsilon, \delta \in \mathbb{R}$. If $f(x)$ is local fractional continuous on $(a, b)$, we denote $f(x) \in C_{\alpha}(a, b)$.

Definition 2.2. [12,13] The local fractional derivative of $f(x)$ of order $\alpha$ at $x=x_{0}$ is defined by

$$
f^{(\alpha)}\left(x_{0}\right)=\left.\frac{d^{\alpha} f(x)}{d x^{\alpha}}\right|_{x=x_{0}}=\lim _{x \rightarrow x_{0}} \frac{\Gamma(\alpha+1)\left(f(x)-f\left(x_{0}\right)\right)}{\left(x-x_{0}\right)^{\alpha}} .
$$

If there exits $f^{(k+1) \alpha}(x)=\overbrace{D_{x}^{\alpha} \cdots D_{x}^{\alpha}}^{n+1 \text { times }} f(x)$ for any $x \in I \subseteq \mathbb{R}$, then we denote $f \in D_{(n+1) \alpha}(I)$, where $n=0,1,2, \cdots$.

Definition 2.3. $[12,13]$ Let $f(x) \in C_{\alpha}[a, b]$. The local fractional integral of function $f(x)$ of order $\alpha$ is defined by

$$
{ }_{a} I_{b}^{\alpha} f(x)=\frac{1}{\Gamma(\alpha+1)} \int_{a}^{b} f(t)(d t)^{\alpha}=\frac{1}{\Gamma(\alpha+1)} \lim _{\Delta t \rightarrow 0} \sum_{j=0}^{N-1} f\left(t_{j}\right)\left(\Delta t_{j}\right)^{\alpha},
$$

where $a=t_{0}<t_{1}<\cdots<t_{N-1}<t_{N}=b,\left[t_{j}, t_{j+1}\right]$ is a partition of the interval $[a, b], \Delta t_{j}=t_{j+1}-t_{j}, \Delta t=$ $\max \left\{\Delta t_{0}, \Delta t_{1} \cdots \Delta t_{N-1}\right\}$.

Note that ${ }_{a} I_{a}^{\alpha} f(x)=0$, and ${ }_{a} I_{b}^{\alpha} f(x)=-{ }_{b} I_{a}^{\alpha} f(x)$ if $a<b$. We denote $f(x) \in I_{x}^{\alpha}[a, b]$ if there exits ${ }_{a} I_{x}^{\alpha} f(x)$ for any $x \in[a, b]$.

Definition 2.4. [23] Let $f: I \subseteq \mathbb{R} \rightarrow \mathbb{R}^{\alpha}$. For any $x_{1}, x_{2} \in I$ and $\lambda \in[0,1]$, if the following inequality

$$
f\left(\lambda x_{1}+(1-\lambda) x_{2}\right) \leq \lambda^{\alpha} f\left(x_{1}\right)+(1-\lambda)^{\alpha} f\left(x_{2}\right),
$$

holds, then $f$ is called a generalized convex function on $I$.

Note that $f(x)=x^{\alpha p}, x \geq 0, p>1$ is a generalized strictly convex function, see [23] and the references therein.

Lemma 2.5. $[12,13]$ If $f(x)=g^{(\alpha)}(x) \in C_{\alpha}[a, b]$, then ${ }_{a} I_{b}^{\alpha} f(x)=g(b)-g(a)$. If $f(x), g(x) \in D_{\alpha}[a, b]$, and $f^{(\alpha)}(x), g^{(\alpha)}(x) \in C_{\alpha}[a, b]$, then

$$
{ }_{a} I_{b}^{\alpha} f(x) g^{(\alpha)}(x)=\left.f(x) g(x)\right|_{a} ^{b}-{ }_{a} I_{b}^{\alpha} f^{(\alpha)}(x) g(x) .
$$

Lemma 2.6. [12, 13]

$$
\begin{aligned}
\frac{d^{\alpha} x^{k \alpha}}{d x^{\alpha}} & =\frac{\Gamma(1+k \alpha)}{\Gamma(1+(k-1) \alpha)} x^{(k-1) \alpha} \\
\frac{1}{\Gamma(\alpha+1)} \int_{a}^{b} x^{k \alpha}(d x)^{\alpha} & =\frac{\Gamma(1+k \alpha)}{\Gamma(1+(k+1) \alpha)}\left(b^{(k+1) \alpha}-a^{(k+1) \alpha}\right) .
\end{aligned}
$$

Lemma 2.7. [13] (Generalized Hölder's inequality) Let $f, g \in C_{\alpha}[a, b], p, q>1$, with $\frac{1}{p}+\frac{1}{q}=1$. Then

$$
\frac{1}{\Gamma(\alpha+1)} \int_{a}^{b}|f(x) g(x)|(d x)^{\alpha} \leq\left(\frac{1}{\Gamma(\alpha+1)} \int_{a}^{b}|f(x)|^{p}(d x)^{\alpha}\right)^{1 / p}\left(\frac{1}{\Gamma(\alpha+1)} \int_{a}^{b}|g(x)|^{q}(d x)^{\alpha}\right)^{1 / q} .
$$




\section{MAIN RESULTS}

First, we establish the following identity.

Lemma 3.1. Let $I \subseteq \mathbb{R}$ be an interval. Let $f: I^{0} \subseteq \mathbb{R} \rightarrow \mathbb{R}^{\alpha}\left(I^{0}\right.$ is the interior of $\left.I\right)$ such that $f \in D_{2 \alpha}\left(I^{0}\right)$ and $f^{(\alpha)} \in C_{\alpha}[a, b]$ for $a, b \in I^{0}$ with $a<b$. Then, for all $x \in[a, b], 0 \leq \lambda \leq 1$, we have the following equality for local fractional integrals:

$$
\begin{aligned}
& \left(\lambda^{\alpha} \Gamma(1+\alpha)-\frac{\Gamma(1+2 \alpha)}{2^{\alpha} \Gamma(1+\alpha)}\right) f\left(\frac{a+b}{2}\right)-\lambda^{\alpha} \Gamma(1+\alpha) \frac{f(a)+f(b)}{2^{\alpha}}+\frac{\Gamma(1+2 \alpha)}{2^{\alpha}(b-a)^{\alpha}} a_{b}^{\alpha} f(x) \\
& =(b-a)^{2 \alpha} \frac{1}{\Gamma(1+\alpha)} \int_{0}^{1} k(t) f^{(2 \alpha)}(t a+(1-t) b)(d t)^{\alpha},
\end{aligned}
$$

where

$$
k(t)= \begin{cases}\frac{t^{\alpha}(t-\lambda)^{\alpha}}{2^{\alpha}}, & 0 \leq t \leq \frac{1}{2} \\ \frac{(1-t)^{\alpha}(1-\lambda-t)^{\alpha}}{2^{\alpha}}, & \frac{1}{2} \leq t \leq 1 .\end{cases}
$$

Proof. Note that

$$
\begin{aligned}
I= & \frac{1}{\Gamma(1+\alpha)} \int_{0}^{1} k(t) f^{(2 \alpha)}(t a+(1-t) b)(d t)^{\alpha} \\
= & \frac{1}{2^{\alpha} \Gamma(1+\alpha)} \int_{0}^{\frac{1}{2}} t^{\alpha}(t-\lambda)^{\alpha} f^{(2 \alpha)}(t a+(1-t) b)(d t)^{\alpha} \\
& +\frac{1}{2^{\alpha} \Gamma(1+\alpha)} \int_{\frac{1}{2}}^{1}(1-t)^{\alpha}(1-\lambda-t)^{\alpha} f^{(2 \alpha)}(t a+(1-t) b)(d t)^{\alpha} \\
= & I_{1}+I_{2} .
\end{aligned}
$$

Using the local fractional integration by parts twice, we deduce

$$
\begin{aligned}
I_{1}= & \frac{1}{2^{\alpha} \Gamma(1+\alpha)} \int_{0}^{\frac{1}{2}} t^{\alpha}(t-\lambda)^{\alpha} f^{(2 \alpha)}(t a+(1-t) b)(d t)^{\alpha} \\
= & \frac{1}{2^{\alpha}(a-b)^{\alpha}}\left[\left.t^{\alpha}(t-\lambda)^{\alpha} f^{(\alpha)}(t a+(1-t) b)\right|_{0} ^{\frac{1}{2}}\right. \\
& \left.-\frac{1}{\Gamma(1+\alpha)} \int_{0}^{\frac{1}{2}}\left(\frac{\Gamma(1+2 \alpha)}{\Gamma(1+\alpha)} t^{\alpha}-\Gamma(1+\alpha) \lambda^{\alpha}\right) f^{(\alpha)}(t a+(1-t) b)(d t)^{\alpha}\right] \\
= & \frac{1}{4^{\alpha}(a-b)^{\alpha}}\left(\frac{1}{2}-\lambda\right)^{\alpha} f^{(\alpha)}\left(\frac{a+b}{2}\right)-\frac{\Gamma(1+2 \alpha)}{2^{\alpha}(a-b)^{\alpha} \Gamma(1+\alpha)} \cdot \frac{1}{\Gamma(1+\alpha)} \int_{0}^{\frac{1}{2}} t^{\alpha} f^{(\alpha)}(t a+(1-t) b)(d t)^{\alpha} \\
& +\frac{\Gamma(1+\alpha)}{2^{\alpha}(a-b)^{\alpha}} \cdot \frac{1}{\Gamma(1+\alpha)} \int_{0}^{\frac{1}{2}} \lambda^{\alpha} f^{(\alpha)}(t a+(1-t) b)(d t)^{\alpha} \\
= & \frac{\left(\frac{1}{2}-\lambda\right)^{\alpha}}{4^{\alpha}(a-b)^{\alpha}} f^{(\alpha)}\left(\frac{a+b}{2}\right)+\frac{f\left(\frac{a+b}{2}\right)}{2^{\alpha}(a-b)^{2 \alpha}}\left(\lambda^{\alpha} \Gamma(1+\alpha)-\frac{\Gamma(1+2 \alpha)}{2^{\alpha} \Gamma(1+\alpha)}\right) \\
& +\frac{\Gamma(1+2 \alpha)}{2^{\alpha}(a-b)^{2 \alpha} \Gamma(1+\alpha)} \int_{0}^{\frac{1}{2}} f(t a+(1-t) b)(d t)^{\alpha}-\frac{\lambda^{\alpha} \Gamma(1+\alpha)}{2^{\alpha}(a-b)^{2 \alpha}} f(b) .
\end{aligned}
$$


In a similar way, we find that

$$
\begin{aligned}
I_{2}= & \frac{1}{2^{\alpha} \Gamma(1+\alpha)} \int_{\frac{1}{2}}^{1}(1-t)^{\alpha}(1-t-\lambda)^{\alpha} f^{(2 \alpha)}(t a+(1-t) b)(d t)^{\alpha} \\
= & -\frac{\left(\frac{1}{2}-\lambda\right)^{\alpha}}{4^{\alpha}(a-b)^{\alpha}} f^{(\alpha)}\left(\frac{a+b}{2}\right)+\frac{f\left(\frac{a+b}{2}\right)}{2^{\alpha}(a-b)^{2 \alpha}}\left(\lambda^{\alpha} \Gamma(1+\alpha)-\frac{\Gamma(1+2 \alpha)}{2^{\alpha} \Gamma(1+\alpha)}\right) \\
& +\frac{\Gamma(1+2 \alpha)}{2^{\alpha}(a-b)^{2 \alpha} \Gamma(1+\alpha)} \int_{\frac{1}{2}}^{1} f(t a+(1-t) b)(d t)^{\alpha}-\frac{\lambda^{\alpha} \Gamma(1+\alpha)}{2^{\alpha}(a-b)^{2 \alpha}} f(a) .
\end{aligned}
$$

Substituting (3.3) and (3.4) into (3.2), we arrive at

$$
\begin{aligned}
I= & I_{1}+I_{2} \\
= & \frac{f\left(\frac{a+b}{2}\right)}{(a-b)^{2 \alpha}}\left(\lambda^{\alpha} \Gamma(1+\alpha)-\frac{\Gamma(1+2 \alpha)}{2^{\alpha} \Gamma(1+\alpha)}\right) \\
& +\frac{\Gamma(1+2 \alpha)}{2^{\alpha}(a-b)^{2 \alpha} \Gamma(1+\alpha)} \int_{0}^{1} f(t a+(1-t) b)(d t)^{\alpha}-\frac{\lambda^{\alpha} \Gamma(1+\alpha)(f(a)+f(b))}{2^{\alpha}(a-b)^{2 \alpha}} .
\end{aligned}
$$

Using the change of the variable $x=t a+(1-t) b, t \in[0,1]$ and multiplying both sides of (3.5) by $(a-$ $b)^{2 \alpha}$, we obtain that

$$
\begin{aligned}
& \left(\lambda^{\alpha} \Gamma(1+\alpha)-\frac{\Gamma(1+2 \alpha)}{2^{\alpha} \Gamma(1+\alpha)}\right) f\left(\frac{a+b}{2}\right)+\frac{\Gamma(1+2 \alpha)}{2^{\alpha}(b-a)^{\alpha} \Gamma(1+\alpha)} \int_{a}^{b} f(x)(d x)^{\alpha} \\
& -\frac{\lambda^{\alpha} \Gamma(1+\alpha)(f(a)+f(b))}{2^{\alpha}} \\
& =(a-b)^{2 \alpha} \frac{1}{\Gamma(1+\alpha)} \int_{0}^{1} k(t) f^{(2 \alpha)}(t a+(1-t) b)(d t)^{\alpha} .
\end{aligned}
$$

Thus the proof is completed .

Theorem 3.2. Let $I \subseteq \mathbb{R}$ be an interval. Let $f: I^{0} \subseteq \mathbb{R} \rightarrow \mathbb{R}^{\alpha}\left(I^{0}\right.$ is the interior of $\left.I\right)$ such that $f \in D_{2 \alpha}\left(I^{0}\right)$ and $f^{(\alpha)} \in C_{\alpha}[a, b]$ for $a, b \in I^{0}$ with $a<b, 0 \leq \lambda \leq 1$. If $\left|f^{(2 \alpha)}\right|$ is generalized convex on $[a, b]$, then, for all $x \in[a, b]$, the following inequalities hold:

$$
\begin{aligned}
& \left|\left(\lambda^{\alpha} \Gamma(1+\alpha)-\frac{\Gamma(1+2 \alpha)}{2^{\alpha} \Gamma(1+\alpha)}\right) f\left(\frac{a+b}{2}\right)-\lambda^{\alpha} \Gamma(1+\alpha) \frac{f(a)+f(b)}{2^{\alpha}}+\frac{\Gamma(1+2 \alpha)}{2^{\alpha}(b-a)^{\alpha}}{ }^{\alpha} I_{b}^{\alpha} f(x)\right| \\
& \leq \begin{cases}(b-a)^{2 \alpha}\left[\frac{\Gamma(1+\alpha)}{\Gamma(1+2 \alpha)}\left(\lambda^{3 \alpha}-\frac{\lambda^{\alpha}}{8^{\alpha}}\right)+\frac{\Gamma(1+2 \alpha)}{\Gamma(1+3 \alpha)}\left(\frac{1^{\alpha}}{16^{\alpha}}-\lambda^{3 \alpha}\right)\right] \\
\times\left(\left|f^{(2 \alpha)}(a)\right|+\left|f^{(2 \alpha)}(b)\right|\right), & 0 \leq \lambda \leq \frac{1}{2}, \\
(b-a)^{2 \alpha}\left[\frac{\lambda^{\alpha}}{8^{\alpha}} \frac{\Gamma(1+\alpha)}{\Gamma(1+2 \alpha)}-\frac{1^{\alpha}}{16^{\alpha}} \frac{\Gamma(1+2 \alpha)}{\Gamma(1+3 \alpha)}\right]\left(\left|f^{(2 \alpha)}(a)\right|+\left|f^{(2 \alpha)}(b)\right|\right), & \frac{1}{2} \leq \lambda \leq 1 .\end{cases}
\end{aligned}
$$


Proof. Using Lemma 3.1 and taking modulus in (3.1), we find that

$$
\begin{aligned}
& \left|\left(\lambda^{\alpha} \Gamma(1+\alpha)-\frac{\Gamma(1+2 \alpha)}{2^{\alpha} \Gamma(1+\alpha)}\right) f\left(\frac{a+b}{2}\right)-\lambda^{\alpha} \Gamma(1+\alpha) \frac{f(a)+f(b)}{2^{\alpha}}+\frac{\Gamma(1+2 \alpha)}{2^{\alpha}(b-a)^{\alpha}} a_{b}^{\alpha} f(x)\right| \\
& \leq(b-a)^{2 \alpha} \frac{1}{\Gamma(1+\alpha)} \int_{0}^{1}|k(t)|\left|f^{(2 \alpha)}(t a+(1-t) b)\right|(d t)^{\alpha} \\
& =\frac{(b-a)^{2 \alpha}}{2^{\alpha}}\left[\frac{1}{\Gamma(1+\alpha)} \int_{0}^{\frac{1}{2}}\left|t^{\alpha}(t-\lambda)^{\alpha}\right|\left|f^{(2 \alpha)}(t a+(1-t) b)\right|(d t)^{\alpha}\right. \\
& \left.\quad+\frac{1}{\Gamma(1+\alpha)} \int_{\frac{1}{2}}^{1}\left|(1-t)^{\alpha}(1-\lambda-t)^{\alpha}\right|\left|f^{(2 \alpha)}(t a+(1-t) b)\right|(d t)^{\alpha}\right] \\
& =\frac{(b-a)^{2 \alpha}}{2^{\alpha}}\left[J_{1}+J_{2}\right] .
\end{aligned}
$$

Assume that $0 \leq \lambda \leq \frac{1}{2}$. Using the generalized convexity of $\left|f^{(2 \alpha)}\right|$ and Lemma 2.6, we get that

$$
\begin{aligned}
J_{1} \leq & \frac{1}{\Gamma(1+\alpha)} \int_{0}^{\frac{1}{2}}\left|t^{\alpha}(t-\lambda)^{\alpha}\right|\left[t^{\alpha}\left|f^{(2 \alpha)}(a)\right|+(1-t)^{\alpha}\left|f^{(2 \alpha)}(b)\right|\right](d t)^{\alpha} \\
= & \frac{1}{\Gamma(1+\alpha)} \int_{0}^{\lambda} t^{\alpha}(\lambda-t)^{\alpha}\left[t^{\alpha}\left|f^{(2 \alpha)}(a)\right|+(1-t)^{\alpha}\left|f^{(2 \alpha)}(b)\right|\right](d t)^{\alpha} \\
& +\frac{1}{\Gamma(1+\alpha)} \int_{\lambda}^{\frac{1}{2}} t^{\alpha}(t-\lambda)^{\alpha}\left[t^{\alpha}\left|f^{(2 \alpha)}(a)\right|+(1-t)^{\alpha}\left|f^{(2 \alpha)}(b)\right|\right](d t)^{\alpha} \\
= & {\left[\frac{\Gamma(1+2 \alpha)}{\Gamma(1+3 \alpha)}\left(2^{\alpha} \lambda^{4 \alpha}-\frac{\lambda^{\alpha}}{8^{\alpha}}\right)+\frac{\Gamma(1+3 \alpha)}{\Gamma(1+4 \alpha)}\left(\frac{1^{\alpha}}{16^{\alpha}}-2^{\alpha} \lambda^{4 \alpha}\right)\right]\left|f^{(2 \alpha)}(a)\right| } \\
& +\left[\frac{\Gamma(1+\alpha)}{\Gamma(1+2 \alpha)}\left(2^{\alpha} \lambda^{3 \alpha}-\frac{\lambda^{\alpha}}{4^{\alpha}}\right)+\frac{\Gamma(1+2 \alpha)}{\Gamma(1+3 \alpha)}\left(1^{\alpha}+\lambda^{\alpha}\right)\left(\frac{1^{\alpha}}{8^{\alpha}}-2^{\alpha} \lambda^{3 \alpha}\right)\right. \\
& \left.+\frac{\Gamma(1+3 \alpha)}{\Gamma(1+4 \alpha)}\left(2^{\alpha} \lambda^{4 \alpha}-\frac{1^{\alpha}}{16^{\alpha}}\right)\right]\left|f^{(2 \alpha)}(b)\right| .
\end{aligned}
$$


Letting $1-t=s$, we also have

$$
\begin{aligned}
J_{2} \leq & \frac{1}{\Gamma(1+\alpha)} \int_{\frac{1}{2}}^{1}\left|(1-t)^{\alpha}(1-\lambda-t)^{\alpha}\right|\left[t^{\alpha}\left|f^{(2 \alpha)}(a)\right|+(1-t)^{\alpha}\left|f^{(2 \alpha)}(b)\right|\right](d t)^{\alpha} \\
= & \frac{1}{\Gamma(1+\alpha)} \int_{\frac{1}{2}}^{1-\lambda}(1-t)^{\alpha}(1-\lambda-t)^{\alpha}\left[t^{\alpha}\left|f^{(2 \alpha)}(a)\right|+(1-t)^{\alpha}\left|f^{(2 \alpha)}(b)\right|\right](d t)^{\alpha} \\
& +\frac{1}{\Gamma(1+\alpha)} \int_{1-\lambda}^{1}(1-t)^{\alpha}(t+\lambda-1)^{\alpha}\left[t^{\alpha}\left|f^{(2 \alpha)}(a)\right|+(1-t)^{\alpha}\left|f^{(2 \alpha)}(b)\right|\right](d t)^{\alpha} \\
= & \frac{1}{\Gamma(1+\alpha)} \int_{\lambda}^{\frac{1}{2}} s^{\alpha}(s-\lambda)^{\alpha}\left[(1-s)^{\alpha}\left|f^{(2 \alpha)}(a)\right|+s^{\alpha}\left|f^{(2 \alpha)}(b)\right|\right](d s)^{\alpha} \\
& +\frac{1}{\Gamma(1+\alpha)} \int_{0}^{\lambda} s^{\alpha}(\lambda-s)^{\alpha}\left[(1-s)^{\alpha}\left|f^{(2 \alpha)}(a)\right|+s^{\alpha}\left|f^{(2 \alpha)}(b)\right|\right](d s)^{\alpha} \\
= & \frac{\Gamma(1+\alpha)}{\Gamma(1+2 \alpha)}\left(2^{\alpha} \lambda^{3 \alpha}-\frac{\lambda^{\alpha}}{4^{\alpha}}\right)+\frac{\Gamma(1+2 \alpha)}{\Gamma(1+3 \alpha)}\left(1^{\alpha}+\lambda^{\alpha}\right)\left(\frac{1^{\alpha}}{8^{\alpha}}-2^{\alpha} \lambda^{3 \alpha}\right) \\
& \left.+\frac{\Gamma(1+3 \alpha)}{\Gamma(1+4 \alpha)}\left(2^{\alpha} \lambda^{4 \alpha}-\frac{1^{\alpha}}{16^{\alpha}}\right)\right]\left|f^{(2 \alpha)}(a)\right| \\
& +\left[\frac{\Gamma(1+2 \alpha)}{\Gamma(1+3 \alpha)}\left(2^{\alpha} \lambda^{4 \alpha}-\frac{\lambda^{\alpha}}{8^{\alpha}}\right)+\frac{\Gamma(1+3 \alpha)}{\Gamma(1+4 \alpha)}\left(\frac{1^{\alpha}}{16^{\alpha}}-2^{\alpha} \lambda^{4 \alpha}\right)\right]\left|f^{(2 \alpha)}(b)\right| .
\end{aligned}
$$

Substituting (3.8) and (3.9) into (3.7), we get

$$
\begin{aligned}
& \left|\left(\lambda^{\alpha} \Gamma(1+\alpha)-\frac{\Gamma(1+2 \alpha)}{2^{\alpha} \Gamma(1+\alpha)}\right) f\left(\frac{a+b}{2}\right)-\lambda^{\alpha} \Gamma(1+\alpha) \frac{f(a)+f(b)}{2^{\alpha}}+\frac{\Gamma(1+2 \alpha)}{2^{\alpha}(b-a)^{\alpha}}{ }^{\alpha} I_{b}^{\alpha} f(x)\right| \\
& \leq(b-a)^{2 \alpha}\left[\frac{\Gamma(1+\alpha)}{\Gamma(1+2 \alpha)}\left(\lambda^{3 \alpha}-\frac{\lambda^{\alpha}}{8^{\alpha}}\right)+\frac{\Gamma(1+2 \alpha)}{\Gamma(1+3 \alpha)}\left(\frac{1^{\alpha}}{16^{\alpha}}-\lambda^{3 \alpha}\right)\right]\left(\left|f^{(2 \alpha)}(a)\right|+\left|f^{(2 \alpha)}(b)\right|\right) .
\end{aligned}
$$

Let $\frac{1}{2} \leq \lambda \leq 1$. Using the generalized convexity of $\left|f^{(2 \alpha)}\right|$ and Lemma 2.6, we get that

$$
\begin{aligned}
J_{1} \leq & \frac{1}{\Gamma(1+\alpha)} \int_{0}^{\frac{1}{2}}\left|t^{\alpha}(t-\lambda)^{\alpha}\right|\left[t^{\alpha}\left|f^{(2 \alpha)}(a)\right|+(1-t)^{\alpha}\left|f^{(2 \alpha)}(b)\right|\right](d t)^{\alpha} \\
= & \frac{1}{\Gamma(1+\alpha)} \int_{0}^{\frac{1}{2}} t^{\alpha}(\lambda-t)^{\alpha}\left[t^{\alpha}\left|f^{(2 \alpha)}(a)\right|+(1-t)^{\alpha}\left|f^{(2 \alpha)}(b)\right|\right](d t)^{\alpha} \\
= & {\left[\frac{\lambda^{\alpha}}{8^{\alpha}} \frac{\Gamma(1+2 \alpha)}{\Gamma(1+3 \alpha)}-\frac{1^{\alpha}}{16^{\alpha}} \frac{\Gamma(1+3 \alpha)}{\Gamma(1+4 \alpha)}\right]\left|f^{(2 \alpha)}(a)\right| } \\
& +\left[\frac{\lambda^{\alpha}}{4^{\alpha}} \frac{\Gamma(1+\alpha)}{\Gamma(1+2 \alpha)}-\frac{1^{\alpha}+\lambda^{\alpha}}{8^{\alpha}} \frac{\Gamma(1+2 \alpha)}{\Gamma(1+3 \alpha)}+\frac{1^{\alpha}}{16^{\alpha}} \frac{\Gamma(1+3 \alpha)}{\Gamma(1+4 \alpha)}\right]\left|f^{(2 \alpha)}(b)\right| .
\end{aligned}
$$


Letting $1-t=s$, we have

$$
\begin{aligned}
J_{2} \leq & \frac{1}{\Gamma(1+\alpha)} \int_{\frac{1}{2}}^{1}\left|(1-t)^{\alpha}(1-\lambda-t)^{\alpha}\right|\left[t^{\alpha}\left|f^{(2 \alpha)}(a)\right|+(1-t)^{\alpha}\left|f^{(2 \alpha)}(b)\right|\right](d t)^{\alpha} \\
= & \frac{1}{\Gamma(1+\alpha)} \int_{\frac{1}{2}}^{1}(1-t)^{\alpha}(t+\lambda-1)^{\alpha}\left[t^{\alpha}\left|f^{(2 \alpha)}(a)\right|+(1-t)^{\alpha}\left|f^{(2 \alpha)}(b)\right|\right](d t)^{\alpha} \\
= & \frac{1}{\Gamma(1+\alpha)} \int_{0}^{\frac{1}{2}} s^{\alpha}(\lambda-s)^{\alpha}\left[(1-s)^{\alpha}\left|f^{(2 \alpha)}(a)\right|+s^{\alpha}\left|f^{(2 \alpha)}(b)\right|\right](d s)^{\alpha} \\
= & {\left[\frac{\lambda^{\alpha}}{4^{\alpha}} \frac{\Gamma(1+\alpha)}{\Gamma(1+2 \alpha)}-\frac{1^{\alpha}+\lambda^{\alpha}}{8^{\alpha}} \frac{\Gamma(1+2 \alpha)}{\Gamma(1+3 \alpha)}+\frac{1^{\alpha}}{16^{\alpha}} \frac{\Gamma(1+3 \alpha)}{\Gamma(1+4 \alpha)}\right]\left|f^{(2 \alpha)}(a)\right| } \\
& +\left[\frac{\lambda^{\alpha}}{8^{\alpha}} \frac{\Gamma(1+2 \alpha)}{\Gamma(1+3 \alpha)}-\frac{1^{\alpha}}{16^{\alpha}} \frac{\Gamma(1+3 \alpha)}{\Gamma(1+4 \alpha)}\right]\left|f^{(2 \alpha)}(b)\right| .
\end{aligned}
$$

Substituting (3.11) and (3.12) into (3.7), we get

$$
\begin{aligned}
& \left|\left(\lambda^{\alpha} \Gamma(1+\alpha)-\frac{\Gamma(1+2 \alpha)}{2^{\alpha} \Gamma(1+\alpha)}\right) f\left(\frac{a+b}{2}\right)-\lambda^{\alpha} \Gamma(1+\alpha) \frac{f(a)+f(b)}{2^{\alpha}}+\frac{\Gamma(1+2 \alpha)}{2^{\alpha}(b-a)^{\alpha}} I_{b}^{\alpha} f(x)\right| \\
& \leq(b-a)^{2 \alpha}\left[\frac{\lambda^{\alpha}}{8^{\alpha}} \frac{\Gamma(1+\alpha)}{\Gamma(1+2 \alpha)}-\frac{1^{\alpha}}{16^{\alpha}} \frac{\Gamma(1+2 \alpha)}{\Gamma(1+3 \alpha)}\right]\left(\left|f^{(2 \alpha)}(a)\right|+\left|f^{(2 \alpha)}(b)\right|\right) .
\end{aligned}
$$

From (3.10) and (3.13), we obtain desired result immediately.

Remark 3.3. If $\alpha=1$ in Theorem 3.2, then (3.6) reduces the inequality in Theorem 1.1.

Corollary 3.4. (Midpoint Inequality) If $\lambda=0$ in Theorem 3.2, we obtain the following inequality

$$
\left|\frac{\Gamma(1+\alpha)}{(b-a)^{\alpha}}{ }_{a} I_{b}^{\alpha} f(x)-f\left(\frac{a+b}{2}\right)\right| \leq \frac{(b-a)^{2 \alpha}}{8^{\alpha}} \frac{\Gamma(1+\alpha)}{\Gamma(1+3 \alpha)}\left(\left|f^{(2 \alpha)}(a)\right|+\left|f^{(2 \alpha)}(b)\right|\right) .
$$

Proposition 3.5. If $\alpha=1$ in Corollary 3.4, we get the following inequality

$$
\left|\frac{1}{b-a} \int_{a}^{b} f(x) d x-f\left(\frac{a+b}{2}\right)\right| \leq \frac{(b-a)^{2}}{48}\left(\left|f^{\prime \prime}(a)\right|+\left|f^{\prime \prime}(b)\right|\right) .
$$

Remark 3.6. We remark here that inequality (3.15) is the same as Proposition 1 in [11].

Corollary 3.7. (Trapezoid Inequality) If $\lambda=1$ in Theorem 3.2, we obtain the following inequality

$$
\begin{aligned}
& \left|\left(\Gamma(1+\alpha)-\frac{\Gamma(1+2 \alpha)}{2^{\alpha} \Gamma(1+\alpha)}\right) f\left(\frac{a+b}{2}\right)-\Gamma(1+\alpha) \frac{f(a)+f(b)}{2^{\alpha}}+\frac{\Gamma(1+2 \alpha)}{2^{\alpha}(b-a)^{\alpha}} a I_{b}^{\alpha} f(x)\right| \\
& \leq(b-a)^{2 \alpha}\left[\frac{1^{\alpha}}{8^{\alpha}} \frac{\Gamma(1+\alpha)}{\Gamma(1+2 \alpha)}-\frac{1^{\alpha}}{16^{\alpha}} \frac{\Gamma(1+2 \alpha)}{\Gamma(1+3 \alpha)}\right]\left(\left|f^{(2 \alpha)}(a)\right|+\left|f^{(2 \alpha)}(b)\right|\right) .
\end{aligned}
$$

Proposition 3.8. If $\alpha=1$ in Corollary 3.7, we obtain the following inequality

$$
\left|\frac{1}{b-a} \int_{a}^{b} f(x) d x-\frac{f(a)+f(b)}{2}\right| \leq \frac{(b-a)^{2}}{24}\left(\left|f^{\prime \prime}(a)\right|+\left|f^{\prime \prime}(b)\right|\right) .
$$

Remark 3.9. We remark here that (3.17) is the same as Proposition 2 in [11]. 
Corollary 3.10. (Simpson Inequality) If $\lambda=\frac{1}{3}$ in Theorem 3.2, we obtain the following inequality

$$
\begin{aligned}
& \left|\left(\frac{\Gamma(1+\alpha)}{3^{\alpha}}-\frac{\Gamma(1+2 \alpha)}{2^{\alpha} \Gamma(1+\alpha)}\right) f\left(\frac{a+b}{2}\right)-\Gamma(1+\alpha) \frac{f(a)+f(b)}{6^{\alpha}}+\frac{\Gamma(1+2 \alpha)}{2^{\alpha}(b-a)^{\alpha}} a_{b}^{\alpha} f(x)\right| \\
& \leq(b-a)^{2 \alpha}\left[\left(\frac{-1}{216}\right)^{\alpha} \frac{\Gamma(1+\alpha)}{\Gamma(1+2 \alpha)}+\left(\frac{11}{432}\right)^{\alpha} \frac{\Gamma(1+2 \alpha)}{\Gamma(1+3 \alpha)}\right]\left(\left|f^{(2 \alpha)}(a)\right|+\left|f^{(2 \alpha)}(b)\right|\right) .
\end{aligned}
$$

Proposition 3.11. If $\alpha=1$ in Corollary 3.10, we get the following inequality

$$
\left|\frac{1}{6}\left[f(a)+4 f\left(\frac{a+b}{2}\right)+f(b)\right]-\frac{1}{b-a} \int_{a}^{b} f(x) d x\right| \leq \frac{(b-a)^{2}}{162}\left(\left|f^{\prime \prime}(a)\right|+\left|f^{\prime \prime}(b)\right|\right) .
$$

Remark 3.12. We remark here that (3.19) is the same as Proposition 3 in [11].

Theorem 3.13. Let $I \subseteq \mathbb{R}$ be an interval. Let $f: I^{0} \subseteq \mathbb{R} \rightarrow \mathbb{R}^{\alpha}$ (I $I^{0}$ is the interior of $\left.I\right)$ such that $f \in$ $D_{2 \alpha}\left(I^{0}\right)$ and $f^{(\alpha)} \in C_{\alpha}[a, b]$ for $a, b \in I^{0}$ with $a<b, 0 \leq \lambda \leq 1$. If $\left|f^{(2 \alpha)}\right|^{q}, q>1$ is generalized convex on $[a, b]$, then, for all $x \in[a, b]$, the following inequalities hold:

$$
\begin{aligned}
& \left|\left(\lambda^{\alpha} \Gamma(1+\alpha)-\frac{\Gamma(1+2 \alpha)}{2^{\alpha} \Gamma(1+\alpha)}\right) f\left(\frac{a+b}{2}\right)-\lambda^{\alpha} \Gamma(1+\alpha) \frac{f(a)+f(b)}{2^{\alpha}}+\frac{\Gamma(1+2 \alpha)}{2^{\alpha}(b-a)^{\alpha}} I_{b}^{\alpha} f(x)\right| \\
& \leq \begin{cases}\frac{(b-a)^{2 \alpha}}{2^{\alpha}}\left(\frac{\Gamma(1+2 \alpha)}{\Gamma(1+3 \alpha)}\left(\frac{1^{\alpha}}{8^{\alpha}}-2^{\alpha} \lambda^{3 \alpha}\right)+\frac{\Gamma(1+\alpha)}{\Gamma(1+2 \alpha)}\left(2^{\alpha} \lambda^{3 \alpha}-\frac{\lambda^{\alpha}}{4^{\alpha}}\right)\right)^{\frac{1}{p}}\left[\left(C_{1}(\lambda, \alpha)\left|f^{(2 \alpha)}(a)\right|^{q}\right.\right. & \\
\left.\left.+C_{2}(\lambda, \alpha)\left|f^{(2 \alpha)}(b)\right|^{q}\right)^{\frac{1}{q}}+\left(C_{1}(\lambda, \alpha)\left|f^{(2 \alpha)}(b)\right|^{q}+C_{2}(\lambda, \alpha)\left|f^{(2 \alpha)}(a)\right|^{q}\right)^{\frac{1}{q}}\right], & 0 \leq \lambda \leq \frac{1}{2}, \\
\frac{(b-a)^{2 \alpha}}{2^{\alpha}}\left(\frac{\lambda^{\alpha}}{4} \frac{\Gamma(1+\alpha)}{\Gamma(1+2 \alpha)}-\frac{\Gamma(1+2 \alpha)}{8^{\alpha} \Gamma(1+3 \alpha)}\right)^{\frac{1}{p}}\left[\left(C_{3}(\lambda, \alpha)\left|f^{(2 \alpha)}(a)\right|^{q}+C_{4}(\lambda, \alpha)\left|f^{(2 \alpha)}(b)\right|^{q}\right)^{\frac{1}{q}}\right. & \\
\left.+\left(C_{3}(\lambda, \alpha)\left|f^{(2 \alpha)}(b)\right|^{q}+C_{4}(\lambda, \alpha)\left|f^{(2 \alpha)}(a)\right|^{q}\right)^{\frac{1}{q}}\right], & \frac{1}{2} \leq \lambda \leq 1 .\end{cases}
\end{aligned}
$$

where $p, q>1$ with $\frac{1}{p}+\frac{1}{q}=1$, and

$$
\begin{aligned}
& C_{1}(\lambda, \alpha)=\frac{\Gamma(1+2 \alpha)}{\Gamma(1+3 \alpha)}\left(2^{\alpha} \lambda^{4 \alpha}-\frac{\lambda^{\alpha}}{8^{\alpha}}\right)+\frac{\Gamma(1+3 \alpha)}{\Gamma(1+4 \alpha)}\left(\frac{1^{\alpha}}{16^{\alpha}}-2^{\alpha} \lambda^{4 \alpha}\right) \\
& C_{2}(\lambda, \alpha)=\frac{\Gamma(1+\alpha)}{\Gamma(1+2 \alpha)}\left(2^{\alpha} \lambda^{3 \alpha}-\frac{\lambda^{\alpha}}{4^{\alpha}}\right)+\frac{\Gamma(1+2 \alpha)}{\Gamma(1+3 \alpha)}\left(1^{\alpha}+\lambda^{\alpha}\right)\left(\frac{1^{\alpha}}{8^{\alpha}}-2^{\alpha} \lambda^{3 \alpha}\right)+\frac{\Gamma(1+3 \alpha)}{\Gamma(1+4 \alpha)}\left(2^{\alpha} \lambda^{4 \alpha}-\frac{1^{\alpha}}{16^{\alpha}}\right) \\
& C_{3}(\lambda, \alpha)=\frac{\lambda^{\alpha}}{8^{\alpha}} \frac{\Gamma(1+2 \alpha)}{\Gamma(1+3 \alpha)}-\frac{1^{\alpha}}{16^{\alpha}} \frac{\Gamma(1+3 \alpha)}{\Gamma(1+4 \alpha)} \\
& C_{4}(\lambda, \alpha)=\frac{\lambda^{\alpha}}{4^{\alpha}} \frac{\Gamma(1+\alpha)}{\Gamma(1+2 \alpha)}-\frac{1^{\alpha}+\lambda^{\alpha}}{8^{\alpha}} \frac{\Gamma(1+2 \alpha)}{\Gamma(1+3 \alpha)}+\frac{1^{\alpha}}{16^{\alpha}} \frac{\Gamma(1+3 \alpha)}{\Gamma(1+4 \alpha)} .
\end{aligned}
$$


Proof. Using Lemma 3.1 and taking modulus in (3.1) yields that

$$
\begin{aligned}
& \left|\left(\lambda^{\alpha} \Gamma(1+\alpha)-\frac{\Gamma(1+2 \alpha)}{2^{\alpha} \Gamma(1+\alpha)}\right) f\left(\frac{a+b}{2}\right)-\lambda^{\alpha} \Gamma(1+\alpha) \frac{f(a)+f(b)}{2^{\alpha}}+\frac{\Gamma(1+2 \alpha)}{2^{\alpha}(b-a)^{\alpha}} a_{b}^{\alpha} f(x)\right| \\
& \leq(b-a)^{2 \alpha} \frac{1}{\Gamma(1+\alpha)} \int_{0}^{1}|k(t)|\left|f^{(2 \alpha)}(t a+(1-t) b)\right|(d t)^{\alpha} \\
& =\frac{(b-a)^{2 \alpha}}{2^{\alpha}}\left[\frac{1}{\Gamma(1+\alpha)} \int_{0}^{\frac{1}{2}}\left|t^{\alpha}(t-\lambda)^{\alpha}\right|\left|f^{(2 \alpha)}(t a+(1-t) b)\right|(d t)^{\alpha}\right. \\
& \left.\quad+\frac{1}{\Gamma(1+\alpha)} \int_{\frac{1}{2}}^{1}\left|(1-t)^{\alpha}(1-\lambda-t)^{\alpha}\right|\left|f^{(2 \alpha)}(t a+(1-t) b)\right|(d t)^{\alpha}\right] .
\end{aligned}
$$

Note that $\frac{1}{p}+\frac{1}{q}=1$, and $\alpha\left(\frac{1}{p}+\frac{1}{q}\right)$ can be written instead of $\alpha$. Using the generalized Hölder inequality, we get

$$
\begin{aligned}
& \left|\left(\lambda^{\alpha} \Gamma(1+\alpha)-\frac{\Gamma(1+2 \alpha)}{2^{\alpha} \Gamma(1+\alpha)}\right) f\left(\frac{a+b}{2}\right)-\lambda^{\alpha} \Gamma(1+\alpha) \frac{f(a)+f(b)}{2^{\alpha}}+\frac{\Gamma(1+2 \alpha)}{2^{\alpha}(b-a)^{\alpha}} a_{b}^{\alpha} f(x)\right| \\
& \leq \frac{(b-a)^{2 \alpha}}{2^{\alpha}}\left[( \frac { 1 } { \Gamma ( 1 + \alpha ) } \int _ { 0 } ^ { \frac { 1 } { 2 } } | t ^ { \alpha } ( t - \lambda ) ^ { \alpha } | ( d t ) ^ { \alpha } ) ^ { \frac { 1 } { p } } \left(\frac{1}{\Gamma(1+\alpha)} \int_{0}^{\frac{1}{2}}\left|t^{\alpha}(t-\lambda)^{\alpha}\right| \mid f^{(2 \alpha)}(t a\right.\right. \\
& \left.\quad+(1-t) b)\left.\right|^{q}(d t)^{\alpha}\right)^{\frac{1}{q}}+\left(\frac{1}{\Gamma(1+\alpha)} \int_{\frac{1}{2}}^{1}\left|(1-t)^{\alpha}(1-\lambda-t)^{\alpha}\right|(d t)^{\alpha}\right)^{\frac{1}{p}} \\
& \left.\quad \times\left(\frac{1}{\Gamma(1+\alpha)} \int_{\frac{1}{2}}^{1}\left|(1-t)^{\alpha}(1-\lambda-t)^{\alpha}\right|\left|f^{(2 \alpha)}(t a+(1-t) b)\right|^{q}(d t)^{\alpha}\right)^{\frac{1}{q}}\right] .
\end{aligned}
$$

Letting $0 \leq \lambda \leq \frac{1}{2}$, we arrive at

$$
\begin{aligned}
& \frac{1}{\Gamma(1+\alpha)} \int_{0}^{\frac{1}{2}}\left|t^{\alpha}(t-\lambda)^{\alpha}\right|(d t)^{\alpha} \\
& =\frac{1}{\Gamma(1+\alpha)} \int_{0}^{\lambda} t^{\alpha}(\lambda-t)^{\alpha}(d t)^{\alpha}+\frac{1}{\Gamma(1+\alpha)} \int_{\lambda}^{\frac{1}{2}} t^{\alpha}(t-\lambda)^{\alpha}(d t)^{\alpha} \\
& =\lambda^{3 \alpha}\left(\frac{\Gamma(1+\alpha)}{\Gamma(1+2 \alpha)}-\frac{\Gamma(1+2 \alpha)}{\Gamma(1+3 \alpha)}\right)+\frac{\Gamma(1+2 \alpha)}{\Gamma(1+3 \alpha)}\left(\frac{1^{\alpha}}{8^{\alpha}}-\lambda^{3 \alpha}\right)+\frac{\Gamma(1+\alpha)}{\Gamma(1+2 \alpha)}\left(\lambda^{3 \alpha}-\frac{\lambda^{\alpha}}{4^{\alpha}}\right) \\
& =\frac{\Gamma(1+2 \alpha)}{\Gamma(1+3 \alpha)}\left(\frac{1^{\alpha}}{8^{\alpha}}-2^{\alpha} \lambda^{3 \alpha}\right)+\frac{\Gamma(1+\alpha)}{\Gamma(1+2 \alpha)}\left(2^{\alpha} \lambda^{3 \alpha}-\frac{\lambda^{\alpha}}{4^{\alpha}}\right) .
\end{aligned}
$$


Since $\left|f^{(2 \alpha)}\right|^{q}$ is generalized convex on $[a, b]$, we find from Lemma 2.6 that

$$
\begin{aligned}
& \frac{1}{\Gamma(1+\alpha)} \int_{0}^{\frac{1}{2}}\left|t^{\alpha}(t-\lambda)^{\alpha}\right|\left|f^{(2 \alpha)}(t a+(1-t) b)\right|^{q}(d t)^{\alpha} \\
\leq & \frac{1}{\Gamma(1+\alpha)} \int_{0}^{\lambda} t^{\alpha}(\lambda-t)^{\alpha}\left[t^{\alpha}\left|f^{(2 \alpha)}(a)\right|^{q}+(1-t)^{\alpha}\left|f^{(2 \alpha)}(b)\right|^{q}\right](d t)^{\alpha} \\
& +\frac{1}{\Gamma(1+\alpha)} \int_{\lambda}^{\frac{1}{2}} t^{\alpha}(t-\lambda)^{\alpha}\left[t^{\alpha}\left|f^{(2 \alpha)}(a)\right|^{q}+(1-t)^{\alpha}\left|f^{(2 \alpha)}(b)\right|^{q}\right](d t)^{\alpha} \\
= & {\left[\frac{\Gamma(1+2 \alpha)}{\Gamma(1+3 \alpha)}\left(2^{\alpha} \lambda^{4 \alpha}-\frac{\lambda^{\alpha}}{8^{\alpha}}\right)+\frac{\Gamma(1+3 \alpha)}{\Gamma(1+4 \alpha)}\left(\frac{1^{\alpha}}{16^{\alpha}}-2^{\alpha} \lambda^{4 \alpha}\right)\right]\left|f^{(2 \alpha)}(a)\right|^{q}+\left[\frac { \Gamma ( 1 + \alpha ) } { \Gamma ( 1 + 2 \alpha ) } \left(2^{\alpha} \lambda^{3 \alpha}\right.\right.} \\
& \left.\left.-\frac{\lambda^{\alpha}}{4^{\alpha}}\right)+\frac{\Gamma(1+2 \alpha)}{\Gamma(1+3 \alpha)}\left(1^{\alpha}+\lambda^{\alpha}\right)\left(\frac{1^{\alpha}}{8^{\alpha}}-2^{\alpha} \lambda^{3 \alpha}\right)+\frac{\Gamma(1+3 \alpha)}{\Gamma(1+4 \alpha)}\left(2^{\alpha} \lambda^{4 \alpha}-\frac{1^{\alpha}}{16^{\alpha}}\right)\right]\left|f^{(2 \alpha)}(b)\right|^{q} \\
= & C_{1}(\lambda, \alpha)\left|f^{(2 \alpha)}(a)\right|^{q}+C_{2}(\lambda, \alpha)\left|f^{(2 \alpha)}(b)\right|^{q} .
\end{aligned}
$$

Letting $1-t=s$, we arrive at

$$
\begin{aligned}
\frac{1}{\Gamma(1+\alpha)} \int_{\frac{1}{2}}^{1}\left|(1-t)^{\alpha}(1-\lambda-t)^{\alpha}\right|(d t)^{\alpha} & =\frac{1}{\Gamma(1+\alpha)} \int_{0}^{\frac{1}{2}}\left|s^{\alpha}(s-\lambda)^{\alpha}\right|(d s)^{\alpha} \\
& =\frac{\Gamma(1+2 \alpha)}{\Gamma(1+3 \alpha)}\left(\frac{1^{\alpha}}{8^{\alpha}}-2^{\alpha} \lambda^{3 \alpha}\right)+\frac{\Gamma(1+\alpha)}{\Gamma(1+2 \alpha)}\left(2^{\alpha} \lambda^{3 \alpha}-\frac{\lambda^{\alpha}}{4^{\alpha}}\right)
\end{aligned}
$$

and

$$
\begin{aligned}
& \frac{1}{\Gamma(1+\alpha)} \int_{\frac{1}{2}}^{1}\left|(1-t)^{\alpha}(1-\lambda-t)^{\alpha}\right|\left|f^{(2 \alpha)}(t a+(1-t) b)\right|^{q}(d t)^{\alpha} \\
= & \frac{1}{\Gamma(1+\alpha)} \int_{0}^{\frac{1}{2}}\left|s^{\alpha}(s-\lambda)^{\alpha}\right|\left|f^{(2 \alpha)}(s b+(1-s) a)\right|^{q}(d s)^{\alpha} \\
\leq & {\left[\frac{\Gamma(1+2 \alpha)}{\Gamma(1+3 \alpha)}\left(2^{\alpha} \lambda^{4 \alpha}-\frac{\lambda^{\alpha}}{8^{\alpha}}\right)+\frac{\Gamma(1+3 \alpha)}{\Gamma(1+4 \alpha)}\left(\frac{1^{\alpha}}{16^{\alpha}}-2^{\alpha} \lambda^{4 \alpha}\right)\right]\left|f^{(2 \alpha)}(b)\right|^{q}+\left[\frac{\Gamma(1+\alpha)}{\Gamma(1+2 \alpha)}\left(2^{\alpha} \lambda^{3 \alpha}-\frac{\lambda^{\alpha}}{4^{\alpha}}\right)\right.} \\
& \left.+\frac{\Gamma(1+2 \alpha)}{\Gamma(1+3 \alpha)}\left(1^{\alpha}+\lambda^{\alpha}\right)\left(\frac{1^{\alpha}}{8^{\alpha}}-2^{\alpha} \lambda^{3 \alpha}\right)+\frac{\Gamma(1+3 \alpha)}{\Gamma(1+4 \alpha)}\left(2^{\alpha} \lambda^{4 \alpha}-\frac{1^{\alpha}}{16^{\alpha}}\right)\right]\left|f^{(2 \alpha)}(a)\right|^{q} \\
= & C_{1}(\lambda, \alpha)\left|f^{(2 \alpha)}(b)\right|^{q}+C_{2}(\lambda, \alpha)\left|f^{(2 \alpha)}(a)\right|^{q} .
\end{aligned}
$$

Substituting (3.22)-(3.25) into (3.21), we obtain the first inequality of (3.2).

On the other hand, letting $\frac{1}{2} \leq \lambda \leq 1$, we have

$$
\begin{aligned}
\frac{1}{\Gamma(1+\alpha)} \int_{0}^{\frac{1}{2}}\left|t^{\alpha}(t-\lambda)^{\alpha}\right|(d t)^{\alpha} & =\frac{1}{\Gamma(1+\alpha)} \int_{0}^{\frac{1}{2}} t^{\alpha}(\lambda-t)^{\alpha}(d t)^{\alpha} \\
& =\frac{\lambda^{\alpha}}{4^{\alpha}} \frac{\Gamma(1+\alpha)}{\Gamma(1+2 \alpha)}-\frac{\Gamma(1+2 \alpha)}{8^{\alpha} \Gamma(1+3 \alpha)} .
\end{aligned}
$$


Since $\left|f^{(2 \alpha)}\right|^{q}$ is generalized convex on $[a, b]$, we obtain from Lemma 2.6 that

$$
\begin{aligned}
& \frac{1}{\Gamma(1+\alpha)} \int_{0}^{\frac{1}{2}}\left|t^{\alpha}(t-\lambda)^{\alpha}\right|\left|f^{(2 \alpha)}(t a+(1-t) b)\right|^{q}(d t)^{\alpha} \\
\leq & \frac{1}{\Gamma(1+\alpha)} \int_{0}^{\frac{1}{2}} t^{\alpha}(\lambda-t)^{\alpha}\left[t^{\alpha}\left|f^{(2 \alpha)}(a)\right|^{q}+(1-t)^{\alpha}\left|f^{(2 \alpha)}(b)\right|^{q}\right](d t)^{\alpha} \\
= & {\left[\frac{\lambda^{\alpha}}{8^{\alpha}} \frac{\Gamma(1+2 \alpha)}{\Gamma(1+3 \alpha)}-\frac{1^{\alpha}}{16^{\alpha}} \frac{\Gamma(1+3 \alpha)}{\Gamma(1+4 \alpha)}\right]\left|f^{(2 \alpha)}(a)\right|^{q} } \\
& +\left[\frac{\lambda^{\alpha}}{4^{\alpha}} \frac{\Gamma(1+\alpha)}{\Gamma(1+2 \alpha)}-\frac{1^{\alpha}+\lambda^{\alpha}}{8^{\alpha}} \frac{\Gamma(1+2 \alpha)}{\Gamma(1+3 \alpha)}+\frac{1^{\alpha}}{16^{\alpha}} \frac{\Gamma(1+3 \alpha)}{\Gamma(1+4 \alpha)}\right]\left|f^{(2 \alpha)}(b)\right|^{q} \\
= & C_{3}(\lambda, \alpha)\left|f^{(2 \alpha)}(a)\right|^{q}+C_{4}(\lambda, \alpha)\left|f^{(2 \alpha)}(b)\right|^{q} .
\end{aligned}
$$

Letting $1-t=s$, we have

$$
\begin{aligned}
\frac{1}{\Gamma(1+\alpha)} \int_{\frac{1}{2}}^{1}\left|(1-t)^{\alpha}(1-\lambda-t)^{\alpha}\right|(d t)^{\alpha} & =\frac{1}{\Gamma(1+\alpha)} \int_{0}^{\frac{1}{2}}\left|s^{\alpha}(s-\lambda)^{\alpha}\right|(d s)^{\alpha} \\
& =\frac{\lambda^{\alpha}}{4^{\alpha}} \frac{\Gamma(1+\alpha)}{\Gamma(1+2 \alpha)}-\frac{\Gamma(1+2 \alpha)}{8^{\alpha} \Gamma(1+3 \alpha)},
\end{aligned}
$$

and

$$
\begin{aligned}
& \frac{1}{\Gamma(1+\alpha)} \int_{\frac{1}{2}}^{1}\left|(1-t)^{\alpha}(1-\lambda-t)^{\alpha}\right|\left|f^{(2 \alpha)}(t a+(1-t) b)\right|^{q}(d t)^{\alpha} \\
= & \frac{1}{\Gamma(1+\alpha)} \int_{0}^{\frac{1}{2}}\left|s^{\alpha}(s-\lambda)^{\alpha}\right|\left|f^{(2 \alpha)}(s b+(1-s) a)\right|^{q}(d s)^{\alpha} \\
\leq & {\left[\frac{\lambda^{\alpha}}{8^{\alpha}} \frac{\Gamma(1+2 \alpha)}{\Gamma(1+3 \alpha)}-\frac{1^{\alpha}}{16^{\alpha}} \frac{\Gamma(1+3 \alpha)}{\Gamma(1+4 \alpha)}\right]\left|f^{(2 \alpha)}(b)\right|^{q} } \\
& +\left[\frac{\lambda^{\alpha}}{4^{\alpha}} \frac{\Gamma(1+\alpha)}{\Gamma(1+2 \alpha)}-\frac{1^{\alpha}+\lambda^{\alpha}}{8^{\alpha}} \frac{\Gamma(1+2 \alpha)}{\Gamma(1+3 \alpha)}+\frac{1^{\alpha}}{16^{\alpha}} \frac{\Gamma(1+3 \alpha)}{\Gamma(1+4 \alpha)}\right]\left|f^{(2 \alpha)}(a)\right|^{q} \\
= & C_{3}(\lambda, \alpha)\left|f^{(2 \alpha)}(b)\right|^{q}+C_{4}(\lambda, \alpha)\left|f^{(2 \alpha)}(a)\right|^{q} .
\end{aligned}
$$

Substituting (3.26)-(3.29) into (3.21), we obtain the second inequality of (3.2). This completes the proof.

Corollary 3.14. (Midpoint Inequality) If $\lambda=0$ in Theorem 3.13, we find the following inequality

$$
\begin{aligned}
& \left|\frac{\Gamma(1+2 \alpha)}{2^{\alpha}(b-a)^{\alpha}} a I_{b}^{\alpha} f(x)-\frac{\Gamma(1+2 \alpha)}{2^{\alpha} \Gamma(1+\alpha)} f\left(\frac{a+b}{2}\right)\right| \\
& \leq \frac{(b-a)^{2 \alpha}}{16^{\alpha}}\left(\frac{\Gamma(1+2 \alpha)}{\Gamma(1+3 \alpha)}\right)^{\frac{1}{p}}\left(\frac{1^{\alpha}}{2^{\alpha}}\right)^{\frac{1}{q}}\left\{\left[\frac{\Gamma(1+3 \alpha)}{\Gamma(1+4 \alpha)}\left|f^{(2 \alpha)}(a)\right|^{q}\right.\right. \\
& \left.\quad+\left(\frac{2^{\alpha} \Gamma(1+2 \alpha)}{\Gamma(1+3 \alpha)}-\frac{\Gamma(1+3 \alpha)}{\Gamma(1+4 \alpha)}\right)\left|f^{(2 \alpha)}(b)\right|^{q}\right]^{\frac{1}{q}} \\
& \left.\quad+\left[\frac{\Gamma(1+3 \alpha)}{\Gamma(1+4 \alpha)}\left|f^{(2 \alpha)}(b)\right|^{q}+\left(\frac{2^{\alpha} \Gamma(1+2 \alpha)}{\Gamma(1+3 \alpha)}-\frac{\Gamma(1+3 \alpha)}{\Gamma(1+4 \alpha)}\right)\left|f^{(2 \alpha)}(a)\right|^{q}\right]^{\frac{1}{q}}\right\},
\end{aligned}
$$


where $p, q>1$ with $\frac{1}{p}+\frac{1}{q}=1$.

Proposition 3.15. If $\alpha=1$ in Corollary 3.14, we get the following inequality

$$
\begin{aligned}
& \left|\frac{1}{b-a} \int_{a}^{b} f(x) d x-f\left(\frac{a+b}{2}\right)\right| \\
& \leq \frac{(b-a)^{2}}{48}\left(\frac{3}{8}\right)^{\frac{1}{q}}\left[\left(\left|f^{\prime \prime}(a)\right|^{q}+\frac{5}{3}\left|f^{\prime \prime}(b)\right|^{q}\right)^{\frac{1}{q}}+\left(\left|f^{\prime \prime}(b)\right|^{q}+\frac{5}{3}\left|f^{\prime \prime}(a)\right|^{q}\right)^{\frac{1}{q}}\right],
\end{aligned}
$$

where $p, q>1$ with $\frac{1}{p}+\frac{1}{q}=1$.

Corollary 3.16. (Trapezoid Inequality) If $\lambda=1$ in Theorem 3.13, we obtain the following inequality

$$
\begin{aligned}
& \left|\left(\Gamma(1+\alpha)-\frac{\Gamma(1+2 \alpha)}{2^{\alpha} \Gamma(1+\alpha)}\right) f\left(\frac{a+b}{2}\right)-\Gamma(1+\alpha) \frac{f(a)+f(b)}{2^{\alpha}}+\frac{\Gamma(1+2 \alpha)}{2^{\alpha}(b-a)^{\alpha}} I_{b}^{\alpha} f(x)\right| \\
& \left.\leq \frac{(b-a)^{2 \alpha}}{8^{\alpha}}\left(\frac{\Gamma(1+\alpha)}{\Gamma(1+2 \alpha)}-\frac{\Gamma(1+2 \alpha)}{2^{\alpha} \Gamma(1+3 \alpha)}\right)\right)^{\frac{1}{p}}\left\{\left[\left(\frac{1^{\alpha}}{2^{\alpha}} \frac{\Gamma(1+2 \alpha)}{\Gamma(1+3 \alpha)}-\frac{1^{\alpha}}{4^{\alpha}} \frac{\Gamma(1+3 \alpha)}{\Gamma(1+4 \alpha)}\right)\left|f^{(2 \alpha)}(a)\right|^{q}\right.\right. \\
& \left.+\left(\frac{\Gamma(1+\alpha)}{\Gamma(1+2 \alpha)}-\frac{\Gamma(1+2 \alpha)}{\Gamma(1+3 \alpha)}+\frac{1^{\alpha}}{4^{\alpha}} \frac{\Gamma(1+3 \alpha)}{\Gamma(1+4 \alpha)}\right)\left|f^{(2 \alpha)}(b)\right|^{q}\right]^{\frac{1}{q}} \\
& \quad+\left[\left(\frac{1^{\alpha}}{2^{\alpha}} \frac{\Gamma(1+2 \alpha)}{\Gamma(1+3 \alpha)}-\frac{1^{\alpha}}{4^{\alpha}} \frac{\Gamma(1+3 \alpha)}{\Gamma(1+4 \alpha)}\right)\left|f^{(2 \alpha)}(b)\right|^{q}\right. \\
& \left.\left.\quad+\left(\frac{\Gamma(1+\alpha)}{\Gamma(1+2 \alpha)}-\frac{\Gamma(1+2 \alpha)}{\Gamma(1+3 \alpha)}+\frac{1^{\alpha}}{4^{\alpha}} \frac{\Gamma(1+3 \alpha)}{\Gamma(1+4 \alpha)}\right)\left|f^{(2 \alpha)}(a)\right|^{q}\right]^{\frac{1}{q}}\right\}
\end{aligned}
$$

where $p, q>1$ with $\frac{1}{p}+\frac{1}{q}=1$.

Proposition 3.17. If $\alpha=1$ in Corollary 3.16, we obtain the following inequality

$$
\begin{aligned}
& \left|\frac{1}{b-a} \int_{a}^{b} f(x) d x-\frac{f(a)+f(b)}{2}\right| \\
\leq & \frac{(b-a)^{2}}{24}\left[\left(\frac{5}{16}\left|f^{\prime \prime}(a)\right|^{q}+\frac{11}{16}\left|f^{\prime \prime}(b)\right|^{q}\right)^{\frac{1}{q}}+\left(\frac{5}{16}\left|f^{\prime \prime}(b)\right|^{q}+\frac{11}{16}\left|f^{\prime \prime}(a)\right|^{q}\right)^{\frac{1}{q}}\right],
\end{aligned}
$$

where $p, q>1$ with $\frac{1}{p}+\frac{1}{q}=1$.

\section{Applications}

We give some applications of the integral inequalities involving the local fractional integral obtained in previous section to obtain estimates of quadrature rules. Consider the division of the interval $[a, b], 0<$ $a<b$, given by $I_{n}: a=x_{0}<x_{1}<\cdots<x_{n}=b$, and the quadrature formula involving the local fractional integral

$$
{ }_{a} I_{b}^{\alpha} f(u)=Q\left(f, I_{n}\right)+E\left(f, I_{n}\right),
$$

where $Q\left(f, I_{n}\right)$ is an approximation of the local fractional integral ${ }_{a} I_{b}^{\alpha} f(u)$, and the remainder term $E\left(f, I_{n}\right)$ is the approximation error. 
Proposition 4.1. Let $I \subseteq \mathbb{R}$ be an interval. Let $f: I^{0} \subseteq \mathbb{R} \rightarrow \mathbb{R}^{\alpha}$ ( $I^{0}$ is the interior of $I$ ) such that $f \in D_{2 \alpha}\left(I^{0}\right)$ and $f^{(\alpha)} \in C_{\alpha}[a, b]$ for $a, b \in I^{0}$ with $a<b$. If $\left|f^{(2 \alpha)}\right|$ is generalized convex on $[a, b]$, then in (4.1), for every division $I_{n}$ of $[a, b]$, the remainder term $E\left(f, I_{n}\right)$ satisfies,

$$
\left|E\left(f, I_{n}\right)\right|=\left|{ }_{a} I_{b}^{\alpha} f(u)-Q\left(f, I_{n}\right)\right| \leq \frac{1}{8^{\alpha} \Gamma(1+3 \alpha)} \sum_{i=0}^{n-1}\left(x_{i+1}-x_{i}\right)^{3 \alpha}\left(\left|f^{(2 \alpha)}\left(x_{i}\right)\right|+\left|f^{(2 \alpha)}\left(x_{i+1}\right)\right|\right),
$$

where

$$
Q\left(f, I_{n}\right)=\frac{1}{\Gamma(1+\alpha)} \sum_{i=0}^{n-1} f\left(\frac{x_{i}+x_{i+1}}{2^{\alpha}}\right)\left(x_{i+1}-x_{i}\right)^{\alpha} .
$$

Proof. Applying Corollary 3.4 to the interval $\left[x_{i}, x_{i+1}\right](i=0, \cdots, n-1)$, we obtain that

$$
\begin{aligned}
& \left|{ }_{x_{i}} I_{x_{i+1}}^{\alpha} f(u)-\frac{1}{\Gamma(1+\alpha)} f\left(\frac{x_{i}+x_{i+1}}{2^{\alpha}}\right)\left(x_{i+1}-x_{i}\right)^{\alpha}\right| \\
& \leq \frac{1}{8^{\alpha} \Gamma(1+3 \alpha)}\left(x_{i+1}-x_{i}\right)^{3 \alpha}\left(\left|f^{(2 \alpha)}\left(x_{i}\right)\right|+\left|f^{(2 \alpha)}\left(x_{i+1}\right)\right|\right) .
\end{aligned}
$$

Summing over $i$ from 0 to $n-1$ and using the triangle inequality, we obtain (4.2).

Next, we consider the applications of our results to the following $\alpha$-type means on fractal sets $(0<$ $\alpha \leq 1)$ :

$$
\begin{gathered}
A(a, b)=\frac{a^{\alpha}+b^{\alpha}}{2^{\alpha}}, \\
L_{n}(a, b)=\left[\frac{\Gamma(1+n \alpha)}{\Gamma(1+(n+1) \alpha)}\left(b^{(n+1) \alpha}-a^{(n+1) \alpha}\right)\right]^{1 / n}, n \in \mathbb{Z} \backslash\{-1,0\}, a, b \in \mathbb{R}, a \neq b .
\end{gathered}
$$

Consider the mapping $f:(0, \infty) \rightarrow \mathbb{R}^{\alpha}, f(x)=x^{n \alpha}, n \in \mathbb{Z} \backslash\{-1,0\}$. Then, $0<a<b$, we have

$$
f\left(\frac{a+b}{2}\right)=[A(a, b)]^{n}, \quad \frac{f(a)+f(b)}{2^{\alpha}}=A\left(a^{n}, b^{n}\right)
$$

and

$$
\frac{1}{(b-a)^{\alpha}} a_{b}^{\alpha} f(x)=\left[L_{n}(a, b)\right]^{n} .
$$

If $n>3$ for function $f(x)=x^{n \alpha}, f:(0, \infty) \rightarrow \mathbb{R}^{\alpha}$, then $\left|f^{(2 \alpha)}\right|$ is a generalized convex function. Using Lemma 2.6, for $0<a<b$, we obtain

$$
\left|f^{(2 \alpha)}(a)\right|+\left|f^{(2 \alpha)}(b)\right|=\frac{\Gamma(1+n \alpha)}{\Gamma(1+(n-2) \alpha)}\left(a^{(n-2) \alpha}+b^{(n-2) \alpha}\right) .
$$

Proposition 4.2. Let $a, b \in R, a<b, 0 \notin[a, b]$ and $n \in \mathbb{Z}, n>3$. Then

$$
\begin{aligned}
& \left|\left(\Gamma(1+\alpha)-\frac{\Gamma(1+2 \alpha)}{2^{\alpha} \Gamma(1+\alpha)}\right)[A(a, b)]^{n}-\Gamma(1+\alpha) A\left(a^{n}, b^{n}\right)+\frac{\Gamma(1+2 \alpha)}{2^{\alpha}}\left[L_{n}(a, b)\right]^{n}\right| \\
& \leq(b-a)^{2 \alpha}\left[\frac{1^{\alpha}}{8^{\alpha}} \frac{\Gamma(1+\alpha)}{\Gamma(1+2 \alpha)}-\frac{1^{\alpha}}{16^{\alpha}} \frac{\Gamma(1+2 \alpha)}{\Gamma(1+3 \alpha)}\right]\left(\frac{\Gamma(1+n \alpha)}{\Gamma(1+(n-2) \alpha)}\left(a^{(n-2) \alpha}+b^{(n-2) \alpha}\right)\right) .
\end{aligned}
$$

Proof. The assertion follows from Corollary 3.7 for $f(x)=x^{n \alpha}, x \in[a, b], n \in \mathbb{Z}, n>3$. 


\section{Acknowledgment}

This paper was supported by the Shaoyang City Science and Technology Plan Project of China under Grants No. 2017GX09 and No. 2018ZD08.

\section{REFERENCES}

[1] E. Set, M.E.Özdemir, S.S. Dragomir, On the Hermite-Hadamard inequality and other integral inequalities involving two functions, J. Inequal. Appl. 2010 (2010), Article ID 148102.

[2] M.A. Latif, Inequalities of Hermite-Hadamard type for functions whose derivatives in absolute value are convex with applications, Arab J. Math. Sci. 21 (2015), 84-97.

[3] M.W. Alomari, M. Darus, U.S. Kirmaci, Some inequalities of Hermite-Hadamard type for $s$-convex functions, Acta Math. Sci. 31B (2011), 1643-1652.

[4] W. Sun, Q. Liu, New Hermite-Hadamard type inequalities for $(\alpha, m)$-convex functions and applications to special means, J. Math. Inequal. 11 (2017), 383-397.

[5] M.A. Latif, M. Shoaib, Hermite-Hadamard type integral inequalities for differentiable m-preinvex and $(\alpha, m)$-preinvex functions, J. Egyptian Math. Soc. 23 (2015), 236-241.

[6] M.E. Özdemir, M. Avci, H. Kavurmaci, Hermite-Hadamardtype inequalities via $(\alpha, m)$-convexity, Comput. Math. Appl. 61 (2011), 2614-2620.

[7] M.E. Özdemir, C. Yıldız, A.O. Akdemir, E. Set, On some inequalities for s-convex functions and applications, J. Inequal. Appl. 2013 (2013), Article ID 333.

[8] Y. Li, T. Du, Some Simpson type integral inequalities for functions whose third derivatives are $(\alpha, m)$-GA-convex functions, J. Egyptian Math. Soc. 24 (2016), 175-180.

[9] L. Chun F. Qi, Inequalities of Simpson type for functions whose third derivatives are extended s-convex functions and applications to means, J. Comput. Anal. Appl. 19 (2015), 555-569.

[10] S. Qaisar, C. He, S. Hussain, A generalizations of Simpsons type inequality for differentiable functions using $(\alpha, m)$ convex functions and applications, J. Inequal. Appl. 2013 (2013), Article ID 158.

[11] M.Z. Sarikaya, N. Aktan, On the generalization of some integral inequalities and their applications, Math. Comput. Modelling 54 (2011), 2175-2182.

[12] X.J. Yang, Advanced Local Fractional Calculus and Its Applications, World Science Publisher, New York, 2012.

[13] X.J. Yang, Local Fractional Functional Analysis and Its Applications, Asian Academic Publisher, Hong Kong, 2011.

[14] J. Choi, E. Set, M. Tomar, Certain generalized Ostrowski type inequalities for local fractional integrals, Commun. Korean Math. Soc., 32 (2017), 601-617.

[15] A. Kiliçmana, W. Salehb, On some inequalities for generalized s-convex functions and applications on fractal sets, J. Nonlinear Sci. Appl. 10 (2017), 583-594.

[16] G. Anastassiou, A. Kashuri, R. Liko, Local fractional integrals involving generalized strongly m-convex mappings, (2018). https://doi.org/10.1007/s40065-018-0214-8.

[17] W Sun, On generalization of some inequalities for generalized harmonically convex functions via local fractional integrals, Quaestiones Math. (2018). https://doi.org/10.2989/16073606.2018.1509242.

[18] W. Sun, Q. Liu, New inequalities of Hermite-Hadamard type for generalized convex functions on fractal sets and its applications, J. Zhejiang Univ. 44 (2017), 47-52.

[19] A. Akkurt, M. Z. Sarikaya, H. Budak, H. Yildirim, Generalized Ostrowski type integral inequalities involving generalized moments via local fractional integrals, Revista de la Real Academia de Ciencias Exactas, Fisicas y Naturales. Serie A. Matematicas, 111 (2017), 797-807.

[20] M.Z. Sarikaya, H. Budak, Generalized Ostrowski type inequalities for local fractional integrals, Proc. Amer. Math. Soc. 145 (2017), 1527-1538.

[21] H. Mo, X. Sui, Hermite-Hadamard-type inequalities for generalized s-convex functions on real linear fractal set $\mathbb{R}^{\alpha}(0<$ $\alpha<1$ ), Math. Sci. 11 (2017), 241-246.

[22] W. Sun, Generalized harmonically convex functions on fractal sets and related Hermite-Hadamard type inequalities, J. Nonlinear Sci. Appl. 10 (2017), 5869-5880. 
[23] H. Mo, X. Sui, D. Yu. Generalized convex functions on fractal sets and two related inequalities, Abst. Appl. Anal. 2014 (2014), Article ID 636751. 